\title{
Impact in Contact Dermatitis during and after SARS-CoV2 Pandemic
}

\author{
Graziella Babino, $M D^{1, *}$ \\ Giuseppe Argenziano, MD, $P h D^{1}$ \\ Anna Balato, MD, PhD ${ }^{1}$
}

\author{
Address \\ *,1Dermatology Unit, Department of Mental and Physical Health and Preventive \\ Medicine, University of Campania "Luigi Vanvitelli", Via Pansini 5, 80131 Naples, Italy \\ Email: graziella.babino@hotmail.it \\ Published online: 10 February 2022 \\ ๑ The Author(s), under exclusive licence to Springer Nature Switzerland AG 2022
}

This article is part of the Topical Collection on Contact Dermatitis

Keywords Contact dermatitis - Prevention - Management - Personal protective equipment - COVID-19 - SARSCoV-2

\begin{abstract}
Purpose of the review Due to the recent COVID-19 pandemic, several skin conditions have emerged due to the preventive measures adopted by both health care workers and the general population against SARS-CoV-2. Above all, wearing of personal protective equipment, frequent hand-washing and disinfecting of surfaces have resulted in an increased risk of irritant or allergic contact dermatitis. The aim of this review is to investigate contact dermatitis associated with COVID-19 pandemic period.

Recent findings There is a real evidence of the rising prevalence of irritant and allergic contact dermatitis in response to the COVID-19 pandemic. The most commonly recorded symptoms are dryness, itch and redness of the skin. Nasal bridge, cheeks, forehead and hands represent the mainly affected skin sites.

Summary Contact dermatitis lesions may appear as a result of various recommendations to prevent transmission of COVID-19. Procedures to alleviate pressure and friction, gentle skin care and adequate moisturizing, have been identified as important preventive strategies for contact dermatitis related to personal protective equipment and personal hygiene measures.
\end{abstract}




\section{Introduction}

On March $11^{\text {th }}$ 2020, coronavirus disease 2019 (COVID-19), due to the novel SARS-CoV-2, was declared a global pandemic by the World Health Organization $[1,2]$. Because of the fast spreading of the contagion, various recommendations to prevent transmission of COVID-19 have been introduced in the general population and particularly among healthcare workers (HCWs), and two efficacious methods include the use of personal protective equipment (PPE) and personal hygiene (PH) measures [3, 4].

On the other hand, prolonged wearing of PPE, frequent hand-washing, and disinfecting of surfaces have been resulted in an increased number of skin diseases. Clinical manifestations include acute and chronic contact dermatitis (CD), secondary infections and

possible aggravation of pre-existing cutaneous disorders such as acne and rosacea [5].

$\mathrm{CD}$ is an inflammatory skin disease characterized by pruritus, erythema, vesicles, and scale. It can present as acute, subacute, or chronic dermatitis. A total of $80 \%$ of CD cases are irritant contact dermatitis (ICD), defined as a localized, non-immunologically driven, inflammatory reaction, resulting from direct cytotoxic effect of a chemical or physical agent. The remaining $20 \%$ of CD cases are allergic contact dermatitis (ACD), a type 4 mediated hypersensitivity to a specific allergen that occurs only in previously sensitized individuals and also develops in a subsequent inflammatory reaction [6].

\section{Contact dermatitis related to personal protective equipment}

PPE includes surgical masks, N-95 respirators, goggles, caps, gloves, and fluidresistant gowns, all of which could be responsible for hyperhydration, friction, epidermal barrier breakdown, and contact reactions, leading to allergic or irritant CD [5]. Of note, erythema, papules, maceration and scaling have been the most commonly reported skin lesions caused by extended wear of PPE among $97 \%$ of HCWs. Clinical manifestations include burning, itching, and stinging. Furthermore, the risk of developing skin damage increase when PPE are worn for more than $6 \mathrm{~h} \mathrm{[7].}$

Facial PPE is one of the most effective methods to prevent the spread of COVID-19 in the hospitals and the community. In addition to masks and respirators, goggles and face shields are other common forms of facial PPE used. The type and the composition of PPE, duration of wear, and underlying skin conditions are all potential factors in the development of CD to facial PPE.

The composition of the facial PPE materials is mainly to consider in suspected cases of ACD, since additives and materials used in their manufacturing process represents the most common cause of ACD $[8,9]$. Textile dyes mix in surgical or cloth masks have primary been reported to lead to ACD [10]. Other identified contact allergens include polyurethanes (toluene-2,4-diisocyanate, 4,4'-diaminodiphenylmethane, hexamethylene diisocyanate) and formaldehyde-releasing preservatives (formaldehyde, 2-bromo-2-nitropropane1,3-diol) [11, 12]. Limited quantity of formaldehyde can as well be detected in polypropylene masks as a degradation by-product [13]. Facial PPE also 
contain potentially allergenic metals such as nickel and cobalt in the nose piece of the mask. Moreover, ACD to facial PPE has been reported to rubber accelerators such as thiurams, carbamates, dialkyl thioureas, and N-isopropyl-N-phenyl-p-phenylendiamine in facemask ear loops [9, 14, 15]. Other potential preservatives include methyldibromoglutanitrile, methyldibromo glutaronitrile, quaternium-15,imidazolinylurea, paraphenylenediamine, and aluminum [16].

Wearing facial PPE for extended periods of time also increases risk of ICD. Friction, pressure, warmth and moisture from respiration may as well induce the development of ICD symptoms, associated to skin barrier dysfunction and skin microbiota alteration.

ICD have been the most widely noted dermatoses (39.5\%) among 43 patients comprising physicians, nurses and paramedical staff involved in managing COVID-19 patients. Goggles led to $51.92 \%$ of facial PPE, followed by N95 respirators $(30.77 \%)$ and face shields (17.31\%). Nasal bridge (63\%) was the commonest anatomical site affected due to dermatoses followed by cheeks and chin (26\%). The duration of wearing the goggles and mask, excessive sweating and ill-fitting masks, all have been associated with increased sensation of irritation, pruritus and erythema [17].

Metin et al. have detected eczema and xerosis in $28 \%$ of HCWs from the use of masks, goggles, and face shields, most frequently involving the nasal bridge, ears, and periocular region [18]. In another study, the use of surgical masks and goggles for $8 \mathrm{~h}$ or more led to skin erosions on the forehead, nasal bridge, and zygoma [19].

One survey has compared the incidences of adverse skin reactions for the mask types used by the general population with a total of 1231 participants involved. The incidence of adverse skin reactions, including ICD, from surgical mask or N95 respirators usage has been higher than that for cloth masks. Pruritis has been the number one complaint among all facial PPE.ICD has been mainly detected in individuals who wore facial PPE for more than $8 \mathrm{~h}$ daily [20].

A study in China has surveyed 61 HCWs who regularly wore N95 respirators for $12 \mathrm{~h}$ a day over an average of 3.5 months: $95.1 \%$ have reported adverse skin reactions to N95s, including nasal bridge scarring (68.9\%) due to pressure from the metal nosepiece, facial itching $(27.9 \%)$, skin damage (26.2\%), dry skin $(24.6 \%)$, and rash (16.4\%) [21 $]$.

Lastly, retroauricular dermatitis following the use of ear loop face masks, composed of latex or thermoelastic polymer, have been noticed in 14 patients, who complained of itching, dryness, scaling, and erythema [22].

Fluid-resistant gowns

There are limited reports of CD associated with prolonged wearing of gowns and coveralls. CD most commonly develops where gowns adhere tightly to the skin, causing heat stress and dehydration. Friction, moisture, and warmth especially in intertriginous areas may increase the risk of ICD [23]. In addition, additive chemicals and dyes fibers used in the manufacturing of gowns including formaldehyde resins and textile dyes can contribute to the 
development of ICD and ACD. [24] One study found that protective clothing and gowns have been identified the top non-glove PPE responsible for ICD [15]. In the Chinese survey previously reported, of the 61 HCWs who regularly wore protective clothing, only $37(60.7 \%)$ have reported adverse skin reactions, including dry skin(36.1\%), itching (34.4\%), rash (11.5\%), and wheals $(3.28 \%)[21 \bullet]$.

\section{Gloves}

Rubber gloves provide an additional layer of protection in preventing viral transmission [25]. On the other hand, excessive use of gloves can lead to a 2.68 times increased risk of developing xerosis and hand eczema [18]. Longterm use of gloves can also paradoxically lead to over hydration of the stratum corneum, with consequent maceration and erosion of the skin [26].

Latex gloves are frequently used among HCWs. Hypersensitivity to natural rubber latex has been increasingly reported, with an incidence of 2.8 to $17 \%$ among HCWs. The most frequently observed reaction has been ICD presenting as dry, crusted, fissuring patches [27]. In the previously mentioned Chinese study, $88.5 \%$ of HCWs wearing rubber latex gloves during the COVID-19 pandemic have complained of skin reactions. The symptoms most commonly reported were dry skin $(55.7 \%)$, itching (31.2\%), rash (24\%), and chapped skin $(21.3 \%)[21 \bullet]$.

Hand dermatitis has been reported with plastic gloves made of nitrile, neoprene, and polyurethane [27]. Primary causes of glove-related ACD have been identified in the rubber gloves accelerators used in the manufacturing of nitrile gloves, such as thiurams, carbamates, diphenylguanidine, mixed dialkyl thioureas, and benzothiazoles, that enlarge the process to synthesize rubber consumer products [28].

At last, some types of powder used in gloves have been associated with an increased risk of $\mathrm{CD}$ due to altered skin $\mathrm{pH}$, and hand eczema has been reported to decrease significantly after using powder-free gloves [27].

\section{Contact dermatitis related to frequent hand-washing and disinfecting of surfaces}

Frequent hand washing, the use of alcohol-based hand sanitizers (ABHS) and the routine use of disinfectant or disinfectant wipes on all contact surfaces have been recommended to decrease the spread of COVID-19 [4].

Soaps and detergents contain fragrances, surfactants, and preservatives that are potential contact allergens leading to ACD [29]. ABHS has been implicated in causing skin dryness and subsequent ICD [30]. Moreover, ABHS often contain ingredients, such as fragrances, tocopherol, propylene glycol, benzoates, and cetylstearyl alcohol, that can also cause ACD [30, 31]. Most disinfectants and disinfectant wipes can be regarded as potential skin irritantsand/or sensitizers, considering their compounds including citric acid, ethyl 
alcohol, hydrogen peroxide, quaternary ammonium, or sodium hypochlorite [32]. Hence, frequent washing and/or hygienization of hands may generate various changes in skin surface, ranging from the development of cutaneous xerosis up to the damage of the skin barrier, leading to ICD or, less commonly, ACD [33]. Furthermore, patients with a personal or family history of atopic dermatitis have a chronically dysfunctional cutaneous barrier that increases their sensitivity to skin irritants.

Hands are affected in greater than $80 \%$ to $90 \%$ occupational CD [34]. The most commonly reported hand symptoms in HCWs during the COVID-19 pandemic have been dryness (92.9\%), itchiness (50\%), and redness (46.4\%) mostly due to hand cleansers followed by the use of disinfectants and gloves [35]. One study demonstrated that hand washing during the COVID-19 pandemic has increased the risk of xerosis and eczema by 3.57 times [18]. Another study showed that hand hygiene measures resulted in a $100 \%$ increase in skin lesions when hygiene routines were performed more than 10 times/day. Moreover, $74.5 \%$ of 526 front-line COVID-19 HCWs reported skin symptoms, including dryness, tenderness, itching, and burning due to excessive hand washing [7]. An observational case series with a total of 72 participants showed that the prevalence of hand ICD in hospitals resulted by an increased frequency in hand washing and the use of ABHS was of $62.5 \%$ [36]. In a german survey, $90.4 \%$ of $114 \mathrm{HCW}$ have reported to have symptoms associated with acute hand dermatitis. Particularly, dryness of hands has been marked in $>80 \%$ of HCWs and about $40 \%$ suffered from erythema. On the contrary, chronic symptoms such as fissures or pain have been noted only in $9.6 \%$ and $4.4 \%$ of all cases, respectively [37].

\section{Prevention and management}

Recommendations for the prevention and management of CD during COVID19 pandemic have been published $[26,38 \bullet \bullet$. Prevention of ICD due to facial PPE relies on strategies to alleviate friction and pressure: thin film dressings, thin hydrocolloid dressings and barrier products made of acrylate, silicon, or dimethicone may be used on pressure or irritated areas, such as the nasal bridge, cheeks, and ears $[8,38 \bullet \bullet, 39]$. Nevertheless, it is crucial to make sure that these skin protectants do not interfere with the fit and safety of the facial PPE, because one recent small study has been analyzed the effect of 5 barrier protectants on $\mathrm{N} 95$ respirator fit. They found fit-testing pass rates have been ranged from 56 to $88 \%$ depending on the skin protectant used; only $36 \%$ of their cohort has been passed with all 5 protectants [40].

Instead of a mask with ear loops, a headband face mask may limit retroauricular irritation and pressure [8]. Furthermore, removing the facial PPE for 5 to 15 min every 2 to $4 \mathrm{~h}$ it has been demonstrated a validate way to relieve pressure on the skin and prevent ICD [41].

Concerning the use of gloves, frequent moisturizing of the hands and using a cotton glove liner can decrease the risk of developing hand ICD [35]. In addition, the use of powder-free gloves has been recommended [27]. In most situations, HCWs have also been encouraged to wear double gloves 
when treating COVID-19 patients: the outer gloves should be at first removed and an accurate hygiene of internal gloves with hydro alcoholic solutions

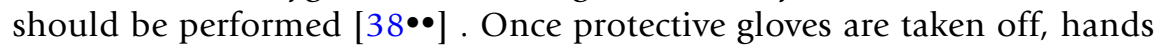
should always be washed with a gentle fragrance-free, hypoallergenic nonsoap skin cleanser, dried softly and moisturized with a hand emollient or protectant.

The only way to prevent ACD is to avoid the relevant contact allergen, which can be identified through patch testing. It is well known that persistent exposure to the allergen can lead to worsening ACD [8]. The American Contact Dermatitis Society has recommended the use of accelerator-free gloves for individuals with suspected or confirmed hand ACD [29]. Indeed, HCWs suffering from hand ACD dramatically improved in terms of disease severity and patient quality of life, after 1-month use of rubber accelerator-free gloves [42]. To avoid $\mathrm{CD}$ as a consequence of frequent hand-washing and disinfecting of surfaces, the use of ABHS, containing at least $60 \%$ ethanol or $70 \%$ isopropyl alcohol and glycerin as moisturizer, has been recommended [3, $38 \bullet \cdot$. In addition, it is required to clean the hands with a gentle hypoallergenic non-soap cleanser and to protect regularly the hands with a fragrancefree, lighter emollient during the day after each hand washing procedure and with a fragrance-free, lipid-rich moisturizer before bedtime [29, 38・•]. Of note, fragrance-free moisturizers with petrolatum or mineral oil have been indicated as the most effective and least allergenic [29].

\section{Conclusions}

The extended use of PPE and enhanced hygiene practices by both HCWs and the general population to prevent transmission of COVID-19, may result in an increased number of skin diseases including $\mathrm{CD}$. In this context, the potential development of $\mathrm{CD}$ is preventable and manageable by the adoption of the appropriate evidence-based skincare protocols. Key factors in the successful prevention and management of CD during COVID-19 pandemic are proper hand hygiene practices, regular application of moisturisers, and avoidance of the known culprit allergen.

\section{Declarations}

\section{Conflict of interest}

Graziella Babino declares that she has no conflict of interest. Giuseppe Argenziano declares that he has no conflict of interest. Anna Balato declares that she has no conflict of interest.

\section{Human and Animal Rights and Informed Consent}

This article does not contain any studies with human or animal subjects performed by any of the authors. 
Papers of particular interest, published recently, have been highlighted as:

- Of importance

$\bullet$ Of major importance

1. WHO Director-General's opening remarks at the media briefing on COVID-19-11 March 2020. Available at:https://www.who.int/dg/speeches/detail/ who-directorgeneral-s-opening-remarks-at-themedia-briefing-oncovid-19.

2. Cucinotta D, Vanelli M. WHO declares COVID-19 a pandemic. Acta Bio-medica: Atenei Parmensis. 2020;91(1):157-60. https://doi.org/10.23750/abm. v91i1.9397.

3. Protect yourself. Available at: https://www.cdc.gov/ coronavirus/2019-ncov/prevent-getting-sick/preve ntion.html.

4. General optimization strategies. Available at: https:// www.cdc.gov/coronavirus/2019-ncov/hcp/ppe-strat egy/general-optimization-strategies.html.

5. Afshar ZM, Babazadeh A, Hasanpour A, Barary M, Sayad B, Janbakhsh A, Aryanian Z, Ebrahimpour $S$. Dermatological manifestations associated with COVID-19: A comprehensive review of the current knowledge. J Med Virol. 2021;93(10):5756-67. https://doi.org/10.1002/jmv.27187.

6. Fonacier L, Bernstein DI, Pacheco K, Holness DL, Blessing-Moore J, Khan D, et al. Contact dermatitis: a practice parameter-update 2015. J Allergy Clin Immunol Pract. 2015;3(3 Suppl):S1-39. https://doi. org/10.1016/j.jaip.2015.02.009.

7. Lan J, Song Z, Miao X, et al. Skin damage among health care workers managing coronavirus disease-2019. J Am Acad Dermatol. 2020;82:1215-6.

8. Yu J, Goldminz A, Chisolm S, et al. Facial personal protective equipment: materials, resterilization methods, and management of occupation-related dermatoses. Dermatitis. 2021;32(2):78-85.

9. Yu J, Chen JK, Mowad CM, et al. Occupational dermatitis to facial personal protective equipmentin health care workers: a systematic review. J Am Acad Dermatol. 2021;84(2):486-94.

10. Mawhirt SL, Frankel D, Diaz AM. Cutaneous manifestations in adult patients with COVID-19 and dermatologic conditions related to the COVID19 pandemic in health care workers. Curr Allergy Asthma Rep. 2020;20(12):75.

11. Xie Z, Yang YX, Zhang H. Mask-induced contact dermatitis in handling COVID-19 outbreak. Contact Dermatitis. 2020;83(2):166-7. https://doi.org/10. 1111/cod.13599.

12. Aerts O, Dendooven E, Foubert K, Stappers S, Ulicki M, Lambert J. Surgical mask dermatitis caused by formaldehyde (releasers)during the COVID-19 pandemic. Contact Dermatitis. 2020;83(2):172-3.
13. Donovan J, Skotnicki-Grant S. Allergic contact dermatitis from formaldehyde textile resins in surgical uniforms and nonwoven textile masks. Dermatitis. 2007;18(1):40-4.

14. Warshaw EM, Schlarbaum JP, Silverberg JI, et al. Safety equipment: when protection becomes a problem. Contact Dermatitis. 2019;81(2):130-2.

15. Bhoyrul B, Lecamwasam K, Wilkinson $M$, et al. A review of non-glove personal protective equipmentrelated occupational dermatoses reported to epiderm between 1993 and 2013. Contact Dermatitis. 2019;80(4):217-21.

16. Bhatia $R$, Sindhuja $T$, Bhatia $S$, et al. Iatrogenic dermatitis in times of COVID-19: a pandemic within a pandemic. J Eur Acad Dermatol Venereol. 2020;34(10):e563-6. https://doi.org/10.1111/jdv. 16710.

17. Singh $\mathrm{M}$, Pawar $\mathrm{M}$, Bothra A, et al. Personal protective equipment induced facial dermatoses in healthcare workers managing coronavirus disease 2019. J Eur Acad Dermatol Venereol. 2020;34(8):e378-80.

18. Metin N, Turan C, Utlu Z. Changes in dermatological complaints among healthcare professionals during the COVID-19 outbreak in Turkey. Acta Dermatovenerol Alp Pannonica Adriat. 2020;29(3):115-22.

19. Zhang B, Zhai R, Ma L. 2019 Novel coronavirus disease epidemic: skin protection for healthcare workers must not be ignored. J Eur Acad Dermatol Venereol. 2020;34(9):e434-5.

20. Chaiyabutr C, Sukakul T, Pruksaeakanan C, et al. Adverse skin reactions following different types of mask usage during the COVID-19 pandemic. J Eur Acad Dermatol Venereol. 2021;35(3):e176-8. https://doi.org/10.1111/jdv.17039.

21. Hu K, Fan J, Li X, et al. The adverse skin reactions of health care workers using personal protective equipment for COVID-19. Medicine (Baltimore). 2020;99(24): e20603. https://doi.org/10.1097/MD. 0000000000020603. (Findings from this study suggest that most health care workers have adverse skin reactions when wearing masks, gloves, and protective clothing for a long period of time.)

22. Bothra A, Das S, Singh M, et al. Retroauricular dermatitis with vehement use of ear loop face masks during COVID-19 pandemic. J Eur Acad Dermatol Venereol. 2020;34(10):e549-52. https://doi.org/10. $1111 /$ jdv. 16692 .

23. Gheisari M, Araghi F, Moravvej H, Tabary M, Dadkhahfar S. Skin reactions to non-glove personal protective equipment: an emerging issue in the 
COVID-19 pandemic. J Eur Acad Dermatol Venereol. 36. 2020;34(7):e297-8. https://doi.org/10.1111/jdv. 16492.

24. Donovan J, Skotnicki-Grant S. Allergic contact dermatitis from formaldehyde textile resins in surgical uniforms and nonwoven textile masks. Dermatitis. 2007;18(1):40-4. https://doi.org/10.2310/6620. 2007.05003.

25. Sanghvi AR. COVID-19: an overview for dermatologists. Int J Dermatol. 2020;59(12):1437-49.

26. Yan Y, Chen H, Chen L, et al. Consensus of Chinese experts on protection of skin and mucous membrane barrier for health-care workers fighting against coronavirus disease 2019. Dermatol Ther. 2020;33(4):1-7.

27. Tabary M, Araghi F, Nasiri S, Dadkhahfar S. Dealing with skin reactions to gloves during the COVID19 pandemic. Infect Control Hosp Epidemiol. 2021;42(2):247-8. https://doi.org/10.1017/ice.2020. 212.

28. Kadivar S, Belsito DV. Occupational dermatitis in health care workers evaluated for suspected allergic contact dermatitis. Dermatitis. 2015;26(4):177-83.

29. Rundle CW, Presley CL, Militello M, et al. Hand hygieneduring COVID-19: recommendations from the American Contact Dermatitis Society. J Am Acad Dermatol. 2020;83(6):1730-7.

30. Voller LM, Schlarbaum JP, Hylwa SA. Allergenic ingredients in health care hand sanitizers in the United States. Dermatitis. 2021;32(3):151-9.

31. Rodriguez-Homs LG, Atwater AR. Allergens in medical hand skin cleansers. Dermatitis. 2019;30(6):336-41.

32. Lachapelle JM. A comparison of the irritant and allergenic properties of antiseptics. Eur J Dermatol. 2014;24:3-9.

33. Long H, Zhao H, Chen A, et al. Protecting medical staff from skin injury/disease caused by personal protective equipment during epidemic period of COVID-19: experience from China. J Eur Acad Dermatol Venereol. 2020;34(5):919-21.

34. Lampel HP, Powell HB. Occupational and hand dermatitis: a practical approach. Clin Rev Allergy Immunol. 2019;56(1):60-71.

35. Alluhayyan OB, Alshahri BK, Farhat AM, et al. Occupational-related contact dermatitis: prevalence and risk factors among healthcare workers in the $\mathrm{Al}^{\prime}$ Qassim region, Saudi Arabia during the COVID19 pandemic. Cureus. 2020;12(10):e10975.
Hadjieconomou S, Hughes J, Kamath S. Occupational skin disease during the COVID-19 pandemic, as captured in a Dermatology staff clinic in the United Kingdom. J Eur Acad Dermatol Venereol. 2020;34(11):e670-1.

37. Guertler A, Moellhoff N, Schenck TL, Hagen CS, Kendziora B, Giunta RE, et al. Onset of occupational hand eczema among healthcare workers during the SARS-CoV-2 pandemic: Comparing a single surgical site with a COVID-19 intensive care unit. Contact Dermatitis. 2020;83(2):108-14.

38.• Balato A, Ayala F, Bruze M, Crepy M-N, Gonçalo M, Johansen J, John SM, Pigatto P, Raimondo A, Rustemeyer T, Schuttelaar M-LA, Svedman C, Aerts O, Uter W, Wilkinson M, Gimenez-Arnau A. European Task Force on Contact Dermatitis statement on coronavirus disease-19 (COVID-19) outbreak and the risk of adverse cutaneous reactions. J Eur Acad Dermatol Venereol. 2020;34(8):e353-4. https://doi.org/10. $1111 / j d v .16557$. (Findings from this manuscript suggest that promoting education on proper use of PPE and on correct hand hygiene could avoid some cutaneous adverse events such as contact dermatitis.)

39. Pacis M, Azor-Ocampo A, Burnett E, et al. Prophylactic dressings for maintaining skin integrity of healthcare workers when using N95 respirators while preventing contamination due to the novel coronavirus: a quality improvement project. J Wound Ostomy Continence Nurs. 2020;47(6):551-7.

40. Bui ATN, Yu Z, Lee $K$, et al. A pilot study of the impact of facial skin protectants on qualitative fit testing of N95 masks. J Am Acad Dermatol. 2021;84(2):554-6.

41. Desai SR, Kovarik C, Brod B, et al. COVID-19 and personal protective equipment: treatment and prevention of skin conditions related to the occupational use of personal protective equipment. J Am Acad Dermatol. 2020;83(2):675-7.

42. Smylie AL, Gill N, Oosterhuis R, et al. Glove induced allergic contact hand dermatitis: a quality improvement initiative. J Cutan Med Surg. 2021;25(2):216-7.

\section{Publisher's Note}

Springer Nature remains neutral with regard to jurisdictional claims in published maps and institutional affiliations. 